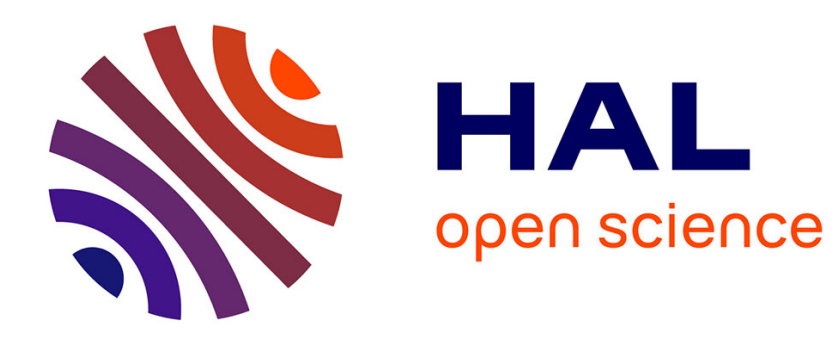

\title{
Numerical aspects of the sweeping process
}

Jean Jacques Moreau

\section{To cite this version:}

Jean Jacques Moreau. Numerical aspects of the sweeping process. Computer Methods in Applied Mechanics and Engineering, 1999, 177, pp.329-349. 10.1016/S0045-7825(98)00387-9 . hal-01349847

\section{HAL Id: hal-01349847 https://hal.science/hal-01349847}

Submitted on 29 Jul 2016

HAL is a multi-disciplinary open access archive for the deposit and dissemination of scientific research documents, whether they are published or not. The documents may come from teaching and research institutions in France or abroad, or from public or private research centers.
L'archive ouverte pluridisciplinaire HAL, est destinée au dépôt et à la diffusion de documents scientifiques de niveau recherche, publiés ou non, émanant des établissements d'enseignement et de recherche français ou étrangers, des laboratoires publics ou privés. 


\title{
Numerical aspects of the sweeping process
}

\author{
J.J. Moreau \\ Laboratoire de Mécanique et Génie Civil, case 048, Université Montpellier II, 34095 Montpellier Cedex 5, France
}

\begin{abstract}
The sweeping process, introduced some time ago by the author with motivation in plasticity theory, today remains an object of mathematical research. It is considered in this paper as the prototype of an evolution conditioned by inequality constraints. Since the governing differential requirements are only of order one with respect to time, this provides a simplified setting for analysing some numerical and theoretical features also present in unilateral dynamics. The latter is governed by differential inclusions of order two, for the numerical handling of which the existing literature proposes diverse strategies, briefly discussed. The paper is especially intended to offer an introduction to the numerical approach called 'contact dynamics'.
\end{abstract}

\section{Introduction}

The numerical dynamics of collections of bodies treated as perfectly indeformable, subject to the constraints of non-interpenetrability, with friction taken into account in the event of contact, is currently an active domain of research. Applications include the dynamics of machines [50], in particular robots, the dynamics of masonry works submitted to transient actions (earthquakes, gusts of wind or impacts) [20], animated computer graphics [4] and the vast area of numerical simulation in granular mechanics $[15,45,46,59]$. In all these applications, assuming the perfect indeformability of each part of the system leads to efficient numerical procedures which, in many circumstances, satisfy the needs. Possibly, some variables are added in order to also account for a certain deformability of these parts (see the contribution of M. Jean in this volume), without essentially changing the computational strategies.

The techniques described in this literature may be classified into three categories.

\section{Event-driven methods}

The methods so qualified are practical, mainly when the concerned time-interval equals the union of not too many subintervals, a priori unknown, over which the status of the various contacts remain unchanged, i.e. no collision which would create new contacts occurs, no contact either gets loose, nor any critical situation needing a change in the analytical expression of the Coulomb law of dry friction is met. On each of these subintervals, the same numerical techniques as in the investigation of machines with classical bilateral, possibly frictional, constraints may be used [18]. As integration proceeds, there is need only to watch the evolution of some indicators. In particular, the contact forces will be calculated. If, after a certain instant, some of the computed values for these forces are found to have directions incompatible with the unilaterality of the non-interpenetrability constraints, the programme decides that the motion has to be otherwise calculated. But one should keep in mind that the contacts which get loose after the critical instant are not necessarily those for which an 
unfeasible contact force has just been evaluated [10]. A popular approach to such discussions consists of reducing them to complementarity problems $[2,4,22,26,50,56]$, similar to what is commonly met in constrained optimization, an aspect that we shall discuss in the sequel. Anyway, as far as it is admitted that contacts involve no adhesion (gluing) effects, determining which of them persist is a mathematical matter.

A much more difficult issue is the calculation of the new velocity in case the critical instant is that of a collision. Only poor phenomenological information is available about the physical circumstances inducing velocity jumps. Even in the simplest case, that of the collision of two otherwise free members of the system, the traditional coefficient of restitution is known to depend not only on the materials these bodies are made of, but also on their shapes and relative orientations at the collision locus [55] (only the case of spherical beads appears relatively comfortable). Worse, if some of the colliding bodies belong to clusters of already contacting system members, percussional reactions should be expected at all existing contact points. Though rigid body collisions are currently an active domain of research (see e.g. [6]), computation has to rely on pragmatic rules whose validity has to be checked in each domain of application.

The event-driven approach becomes much simpler when the assumption is made that interactions only consist of instant collisions, between which each body moves in free ballistic flight. This may prove realistic in the dynamics of loose granulates [47], but here again, sufficiently reliable collision laws are wanted. Incidentally, a trick to simulate persistent contact in this line consists of introducing an artificial agitation [16].

\section{- Smoothing methods}

'Nonsmoothness' is the salient feature of the problems in view. In fact, after the set of possible positions of the investigated system has been parametrized through an element $q$ of $\boldsymbol{R}^{n}$, the geometric restriction that the non-interpenetrability constraints impose on $q$ are expressed by a set of inequalities. Hence, instead of running in a smooth submanifold, as in traditional analytical dynamics, the point $q$ is confined in a region of $\boldsymbol{R}^{n}$ whose boundary is made of a lot of pieces of hypersurfaces (millions or billions of them in current applications to granular materials): this is nonsmoothness in space. Furthermore, collisions are expected to induce velocity jumps: this is nonsmoothness in time. To end, the contact forces or 'reactions' associated with the noninterpenetrability constraints are governed by highly irregular laws. These forces vanish as soon as the corresponding contacts break while, if contact holds, the commonly stipulated mechanical conditions do not express them as functions of $q$. If, in addition, dry friction is taken into account (most usually in the form of Coulomb's law), it introduces some irregular relationships between contact forces and the sliding velocities. All this may be called nonsmoothness in law.

In such a state of affairs, a natural move is to replace, approximately, the nonsmooth governing relationships by some regularized ones. First, the non-interpenetrability constraints will be replaced by some stiff repulsion laws which take effect as soon as two members of the system come close to each other. This automatically handles the possible collisions, as far as one considers them as 'elastic', while the dissipativity of collisions may be accounted for by adding some damping actions or also by using different repulsion laws in the episodes of approach and of separation [15]. Similarly, frictional contact may be somewhat regularized through the introduction [9] of local elastic micro-deformation (in favour of which some experimental evidences exist [48]) and of viscosity-like effects. The dynamics of the approximate system is then governed by differential equations with sufficient regularity to be handled through standard numerical techniques. The drawback is that the need for precision requires the use of very stiff appproximate laws. Hence, the time-stepping procedures applied have to resort to very small step-length and possibly also have to enforce numerical stability by introducing artificial damping or artificially increasing inertia. When treating dynamical applications, the effect of such an artificial alteration of the mechanical data may blur the picture. Significant simulations of loose (collisional) flows of granular materials have been obtained in that way, but when dense collections of bodies are concerned (pieces of masonry or compact granulates) the method is mainly applied to quasi-static evolutions in which only a succession of equilibrium states is looked for. Dynamical computation is then used only as a way of attaining each of these equilibrium states, a strategy referred to as 'dynamic relaxation'.

The pioneering work of Cundall [8] was precisely based on the regularization strategy, today implemented in the majority of commercial pieces of software intended to handle non-interpenetrability. The denominations of 'discrete element methods' or 'distinct element methods' (DEM) are commonly used to refer to this approach. Also, because the computation techniques applied in such implementations are close to those of molecular 
simulations, the denomination of 'molecular dynamics' (MD) method is also used, specially in the domain of granular mechanics [59].

\section{- Contact dynamics}

This is the method advocated by the author, to which the present paper is meant to provide an introduction. It originated from Moreau [42,43], where the (unilateral) contact, possibly frictional and/or collisional, between rigid bodies received a formulation in terms of elementary convex analysis which proves suitable for computation. Mathematically, the resulting evolution problems are governed in smooth cases by differential inclusions. General information on the latter concept may be found in [3,11], but the need for also treating non-smooth evolutions calls for its extension to that of a measure differential inclusion. The sweeping process introduced earlier by the author, with motivation in the quasi-static evolution of elastoplastic systems $[34,35,38]$ seems to have provided the first occurence of measure differential inclusions in literature. This process and some related evolution problems are still today the object of mathematical research $[17,24,25]$. In the present paper, the basic facts of its theory have been estimated sufficiently clear to make a tutorial introduction to the specificities of the handling of unilaterality in mechanics.

The directing idea of the contact dynamics approach is that the main object of computation is the velocity function $t \mapsto u \in \boldsymbol{R}^{n}$. Time-stepping algorithms essentially have to determine the evolution of this function, by applying the principles of dynamics and the specified force laws. The position function $t \mapsto q$ is only to be updated at each step through adequate integration (possibly also, some members of $u$ are 'pseudo-parameters' such as the components of the spin vector of a solid, instead of the time-derivatives of position parameters). From the geometrical standpoint, one should observe that, in analytical dynamics, the position $q$ ranges in some differential manifold, while for every $q$ the possible velocities are elements of the tangent space to the manifold at this point. The latter is a vector space relative to such concepts as linearity, convexity, etc. make sense, while no algebraic concept of this sort is generally available in the position manifold.

Contact dynamics procedures rest on drawing the balance of momentum of the investigated mechanical system over each time-step. No estimation of the acceleration is needed and the burden of mathematically calculating the curvatures of the involved surfaces is avoided. These curvatures are simply accounted for by that, from one step to the other, the normal directions to the detected contacts vary. The method results in time-stepping schemes which, at least in what concerns the velocity function, are of the implicit type. Recently, several authors have undertaken the treatment of multibody dynamics in similar lines [2,53,54].

In principle, the contact dynamics algorithms are ready at each step to treat collisions on the same footing as persistent contacts but, of course, the need of physical information about such phenomena is not overcome.

\section{The sweeping process}

\subsection{Definition}

Let $H$ be a real Hilbert space and let $I$ be a real interval, viewed here as an interval of time, containing its origin $t_{0}$. A moving set is given, i.e. a multifunction $t \mapsto C(t)$ (this is also called a set-valued mapping). It is assumed, for every $t$ in $I$, that $C(t)$ is a nonempty closed convex subset of $H$.

For $x$ in $H$ and for an arbitrary convex subset $C$ of $H$ one denotes by $N_{C}(x)$ the (outward) normal cone to $C$ at point $x$, in the standard sense of convex analysis, namely with the dot denoting the scalar product in $H$,

$$
N_{C}(x):= \begin{cases}\{y \in H \mid \forall \xi \in C:(\xi-x) . y \leq 0\} & \text { if } x \in C \\ \emptyset & \text { otherwise. }\end{cases}
$$

Recall that, except when specified differently, by a cone is meant a homogeneous one, i.e. with the zero of $H$ as apex.

If $x \in C$, the cone $N_{C}(x)$ contains at least the zero of $H$ and, in particular, reduces to this single element if $x$ happens to be an internal point of $C$. If $x$ lies at a regular point of the boundary of $C$, the cone consists of the 
half-line emanating from the zero of $H$, directed as the outward normal to $C$ at $x$. That $N_{C}(x)$ should be empty if $x \notin C$ is not an ad hoc trick of notation, but is consistent with the general concepts of the "subdifferential calculus' $[19,32,51]$.

By a strong solution of the sweeping process by the moving set $C$ over the time-interval $I$, one means a function $u: I \rightarrow H$, locally absolutely continuous (i.e. absolutely continuous on every compact subinterval of $I$ ) verifying for every $t$ in $I$ the differential inclusion

$$
-\frac{\mathrm{d} u}{\mathrm{~d} t} \in N_{C(t)}(u(t))
$$

Recall that the assumption of local absolute continuity for $u$ implies the existence of a function $\dot{u}$ with values in $H$, defined in $I$ with the possible exception of a Lebesgue-null set $\mathcal{N}$ and locally Lebesgue-integrable, such that

$$
\forall t \in I: \quad u(t)=u\left(t_{0}\right)+\int_{t_{0}}^{t} \dot{u}(s) \mathrm{d} s .
$$

By $\mathrm{d} u / \mathrm{d} t$ in the left-hand side of $(1)$, we mean $\dot{u}(t)$; the function $\dot{u}$ may be extended to $\mathcal{N}$ with zero value, an extension which trivially satisfies (1).

This conception of the derivative of a function as 'what allows one to retrieve the function through integration' reflects the familiar approach to existence questions for ordinary differential equations, namely seeking fixed points of integral operators. In our view, this practice meets most of the needs in mechanics, possibly by resorting to other 'base' measures than Lebesgue as we are to show later. In addition, one has to recall that, for almost every $t$, the function $u$ possesses a derivative in the local sense, equal to $\dot{u}(t)$. All these are standard facts of integration theory when real or $\boldsymbol{R}^{n}$-valued functions are concerned. For their extension to functions with values in a reflexive Banach space such as $H$ here, one may refer to [13,14].

Inclusion (1) ensures that $u(t) \in C(t)$ since otherwise the right-hand side would be empty. If, for $t$ ranging in some subinterval, $u(t)$ happens to lie in the possible interior of $C(t)$, this point remains at rest since $N_{C(t)}(u(t))$ then reduces to $\left\{0_{H}\right\}$. It is only when caught-up with by the boundary of $C(t)$ that the point takes a motion, in normal inward direction, so as to go on belonging to $C(t)$.

The convexity of a set $C$ classically implies that the multifunction $x \mapsto N_{C}(x)$ is monotone in the sense of Minty [5], i.e. if $x_{1}, x_{2}$ are elements of $H$, if $y_{1} \in N_{C}\left(x_{1}\right)$ and $y_{2} \in N_{C}\left(x_{2}\right)$ one has,

$$
\left(x_{2}-x_{1}\right) \cdot\left(y_{2}-y_{1}\right) \geqslant 0 \text {. }
$$

Let $t \mapsto u_{1}(t)$ and $t \mapsto u_{2}(t)$ be two functions verifying (1). Through the expression of the derivative of the real function $t \mapsto\left\|u_{2}(t)-u_{1}(t)\right\|^{2}$, property (2) readily implies that this function is nonincreasing; in other words, the flow of the possible solutions to the differential inclusion (1) is non-expansive. Consequently, in spite of the right-hand side of (1) being multivalued, at most one solution can agree with an initial condition $u\left(t_{0}\right)=u_{0}$. The existence question, which of course is conditioned by some regularity assumption regarding the motion of $C$, will shortly be reviewed in Section 5 .

If the assumption of the convexity of $C$ is dropped, some other definitions of the normal cone remain available (we shall come back to this in Section 7), giving a more general scope to the above differential inclusion [57,58]. Short of property (2), the uniqueness of solution to the initial value problem may fail. In this connection, let us mention another mechanical image of the sweeping process, suggestive when $\operatorname{dim} H=2$.

Picture the product $I \times H$ as the physical three-dimensional space, with $I$ corresponding to the vertical axis oriented downward. Imagine the set $\{(t, x) \in I \times H \mid x \in C(t)\}$ as an underground cavity and the curve $\{(t, x) \in I \times H \mid x=u(t)\}$, namely the graph of the unknown function $u$, as a tiny waterstream falling down this cavity. Condition (1) is equivalent to the following statements: (i) any arc of this stream which happens to be detached from the cavity wall is rectilinear and vertical; (ii) when water is running over the wall, it follows a line orthogonal to the level curves of the wall surface, i.e. a line of steepest descent; this agrees with hydrodynamics under the simplifying assumption that inertia may be neglected, comparative to wall friction and gravity; and (iii) the minus sign in (1) precisely expresses that such a contact with the wall may take place only on a part of this wall exposed upward; if it crosses the rim of a possible overhang, water gets loose from the surface and falls vertically down, as described in (i). 


\subsection{More about convex cones}

Let the polar cone of an arbitrary cone $P \subset H$ be defined as

$$
P^{*}:=\{y \in H \mid \forall x \in P: x . y \leqslant 0\} .
$$

This is a closed convex cone in $H$. If the cone $P$ itself is closed and convex, one proves that it equals in turn the polar cone of $P^{*}$, making what is commonly called a pair of dual cones.

Dual cones provide a vivid setting to examplify some duality features of what is called "quadratic programming', i.e. the minimization of quadratic functions over convex sets, and in the same line of thought the equivalence of a pair of dual problems to what is usually named a 'complementarity problem'. The specific quadratic programme considered here is to determine, in a nonempty closed convex subset $C$ of our Hilbert space $H$, the nearest point to a given $z \in H$, that we shall denote by $\operatorname{prox}(C, z)$. In fact, this determination amounts to minimizing in $C$ the quadratic function $x \mapsto\|x-z\|^{2}$.

For every $z$ in $H$, the existence and uniqueness of $x:=\operatorname{prox}(C, z)$ is classical, as well as the following characterization of this point

$$
z-x \in N_{C}(x) \text {. }
$$

The dual cone decomposition lemma [30,32] reads:

Let $P$ and $Q$ denote a pair of dual cones in the Hilbert space $H$. For three elements $x, y, z$ of $H$, the statements (4) and (5) are equivalent.

$$
\begin{aligned}
& z=x+y, \quad x \in P, \quad y \in Q, \quad x . y=0 . \\
& x=\operatorname{prox}(P, z), \quad y=\operatorname{prox}(Q, z) .
\end{aligned}
$$

Among the numerous elementary consequences of this lemma, let us observe this. In view of (4), one has the equality $\|z\|^{2}=\|x\|^{2}+\|y\|^{2}$, through which any upper bound of the distance of $z$ to one of the two sets $P$ and $Q$ generates a lower bound of the distance to the other.

One also readily derives from the lemma a characterization of the normal cone to one of the cones $P$ or $Q$ at any point. In fact, by taking (3) into account, one finds for every $x$

$$
y \in N_{P}(x) \Leftrightarrow y \in Q \text { and } x . y=0
$$

and symmetrically. In particular, this shows that $N_{P}(x) \subset Q$ and $N_{P}\left(0_{H}\right)=Q$.

\section{Two examples of moving convex sets}

\subsection{A set moving by translation}

Let $C_{0}$ be a fixed closed convex subset of $H$ and let $a: I \rightarrow H$ be given, locally absolutely continuous. Define $C(t)=C_{0}+a(t)$ and consider a solution $u$ to (1) in this case.

For every $t$ in $I$, the moving point $w(t):=u(t)-a(t)$ remains in $C_{0}$. Let $t_{1} \in I$; the definition of the normal cone to $C_{0}$ at point $w\left(t_{1}\right)$ makes that

$$
\forall t \in I, \quad \forall \nu \in N_{C_{0}}\left(w\left(t_{1}\right)\right):\left(w(t)-w\left(t_{1}\right)\right) . \nu \leq 0 .
$$

In other words, $w(t)-w\left(t_{1}\right)$ belongs to the polar cone $N_{C_{0}}^{*}\left(w\left(t_{1}\right)\right)$, hence the same is true for the right-derivative

$$
\dot{w}^{+}\left(t_{1}\right):=\lim _{t \downarrow_{t_{1}}} \frac{w(t)-w\left(t_{1}\right)}{t-t_{1}}
$$

if it exists. Generally speaking, $N_{C_{0}}^{*}(x)$ is called the tangent cone to $C_{0}$ at point $x$. 
The left-derivative $\dot{w}^{-}\left(t_{1}\right)$ is symmetrically found to belong to $-N_{C_{0}}^{*}\left(w\left(t_{1}\right)\right)$, hence the (bilateral) derivative $\dot{w}\left(t_{1}\right)$, if it exists, belongs to

$$
L_{C_{0}}\left(w\left(t_{1}\right)\right):=N_{C_{0}}^{*}\left(w\left(t_{1}\right)\right) \cap-N_{C_{0}}^{*}\left(w\left(t_{1}\right)\right),
$$

a linear subspace of $H$ called the tangent space to $C_{0}$ at point $w\left(t_{1}\right)$ (possibly reduced to the zero of $H$ ).

The above are merely kinematical consequences of $u(t)$ belonging to $C(t)$ for every $t$. Taking into account the governing condition (1) of the sweeping process and the obvious equality of the respective normal cones at the corresponding points of translate sets, one has $-\dot{u}\left(t_{1}\right) \in N_{C_{0}}\left(w\left(t_{1}\right)\right)$. Clearly, all elements of the set $N_{C_{0}}\left(w\left(t_{1}\right)\right)$ are orthogonal to $L_{C_{0}}\left(w\left(t_{1}\right)\right)$, hence at every instant $t_{1}$ such that the considered derivatives exist (bilaterally), the scalar product $\dot{u} \cdot \dot{w}$ vanishes, i.e. $\dot{u} \cdot(\dot{a}-\dot{u})=0$. By putting this equality together with the inclusions $\dot{u} \in-N_{C}$ and $\dot{a}-\dot{u} \in-N_{C}^{*}$, one brings to light the familiar ternary pattern of a system of complementarity conditions in precisely the form displayed in Subsection 2.2. Here, $-N_{C}$ and $-N_{C}^{*}$ are dual cones, therefore the dual cone decomposition lemma yields

$$
\begin{aligned}
& \dot{u}=\operatorname{prox}\left(-N_{C}, \dot{a}\right), \\
& \dot{a}-\dot{u}=\operatorname{prox}\left(-N_{C}^{*}, \dot{a}\right) .
\end{aligned}
$$

By using translation and symmetry about the origin, one transforms (8) into

$$
\dot{u}=\operatorname{prox}\left(\dot{a}+N_{C}^{*}, 0_{H}\right) \text {, }
$$

i.e. $\dot{u}$ equals the element of minimal norm in $\dot{a}+N_{C}^{*}$. In the foregoing, what was said of the motion of $w=u-a$ in the fixed set $C_{0}$, implies that $\dot{a}+N_{C}^{*}$ equals the set of the values of the right-derivative $\dot{u}^{+}$ kinematically compatible with $u(t)$ remaining in $C(t)$ for every $t$. We shall refer to this set as the admissible right-velocity set, (ARVS).

As for (7), it implies, in particular, the inequality $\|\dot{i}\| \leq\|\dot{a}\|$. The definition of the moving set $C(t)$ as the translate $C_{0}+a(t)$ allows one in the present case to interpret the sweeping process as a driving mechanism tending to make $u$ accompany the given motion of $a$, but suffering some clearance. The above inequality expresses that the instant speed of $u$ cannot exceed that of $a$ and, by integration over a time-interval, that the length of the path of $u$ cannot exceed that of the path of $a$. Establishing a priori some inequalities of this sort plays a central role in existence proofs.

\subsection{Finite intersection of smoothly moving regions}

Let us now consider the case of a subset $C(t)$ of $H$ defined by a finite collection of inequalities

$$
C(t)=\left\{x \in H \mid c_{\alpha}(t, x) \leqslant 0, \alpha=1,2, \ldots, \kappa\right\},
$$

where the functions $c_{\alpha}: I \times H \rightarrow \boldsymbol{R}$ are continuously differentiable, convex in the argument $x$. Their $x$-gradients, elements of $H$ denoted in the sequel by $\nabla c_{\alpha}$, are supposed nonzero at least in neighbourhoods of the respective hypersurfaces $c_{\alpha}=0$. At a fixed $t$, the convexity of $c_{\alpha}$ classically implies that, for every $x$ and $u$ in $H$, the inequality

$$
(x-u) . \nabla c_{\alpha}(t, u) \leqslant c_{\alpha}(t, x)-c_{\alpha}(t, u)
$$

holds. For every $t$ and $u$, put

$$
K(t, u):=\left\{\alpha \in\{1,2, \ldots, \kappa\} \mid c_{\alpha}(t, u)=0\right\} .
$$

Inequality (11) yields that, for every $\alpha$ in $K(t, u)$, the element $\nabla c_{\alpha}(t, u)$ belongs to the normal cone $N_{C(t)}(u)$. Whether the totality of this normal cone is generated by nonnegative combination of the said elements is an issue of the 'subdifferential calculus' [19,51]. We shall assume in the sequel that $N_{C(t)}(u)$ possesses this generation property. A standard sufficient condition is that $C(t)$ possess a nonempty interior.

Define

$$
W(t, u):=\left\{w \in H \mid \forall \alpha \in K(t, u): \partial c_{\alpha} / \partial t+w \cdot \nabla c_{\alpha} \leq 0\right\},
$$

a closed convex polyhedral subset of $H$ (in particular this equals the whole of $H$ if $K(t, u)=\emptyset$ ). 
Let $t \mapsto u(t)$ denote a moving point belonging to $C(t)$ for every $t$ and let $t_{1} \in I$. If $\alpha \in K\left(t_{1}, u\left(t_{1}\right)\right)$, the right derivative at point $t_{1}$ of the real function $t \mapsto c_{\alpha}(t, u(t))$, if this derivative exists, is necessarily $\leqslant 0$. Using the chain rule, one concludes that the right derivative $\dot{u}^{+}\left(t_{1}\right)$, if it exists, belongs to $W\left(t_{1}, u\left(t_{1}\right)\right)$. The latter set constitutes in the present case the ARVS.

Symmetrically, the left derivative $\dot{u}^{-}\left(t_{1}\right)$, if it exists, belongs to the set defined by replacing $\leqslant 0$ by $\geqslant 0$ in (13), hence, if the (bilateral) derivative $\dot{u}\left(t_{1}\right)$ exists, it verifies

$$
\forall \alpha \in K\left(t_{1}, u\left(t_{1}\right)\right): \quad \partial c_{\alpha} / \partial t+\dot{u}\left(t_{1}\right) \cdot \nabla c_{\alpha}=0
$$

By substracting these equalities from the respective inequalities involved in the definition (13), one sees that, in the present circumstance, $W\left(t_{1}, u\left(t_{1}\right)\right)$ is a (non homogeneous) cone admitting $\dot{u}\left(t_{1}\right)$ as apex. Up to the translation $-\dot{u}\left(t_{1}\right)$ in $H$, this equals the polar cone of the cone generated by the elements $\nabla c_{\alpha}\left(t_{1}, u\left(t_{1}\right)\right), \alpha \in K\left(t_{1}, u\left(t_{1}\right)\right)$, namely $N_{C\left(t_{1}\right)}\left(u\left(t_{1}\right)\right)$ in view of the assumed generation property. Owing to $(6)$, this also equals the normal cone

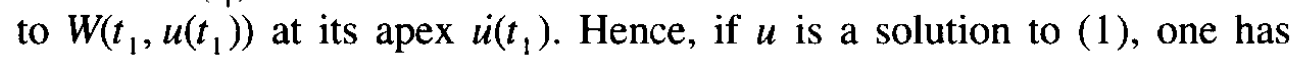

$$
0_{H}-\dot{u}\left(t_{1}\right) \in N_{W\left(t_{1}, u\left(t_{1}\right)\right)}\left(\dot{u}\left(t_{1}\right)\right) \text {. }
$$

Due to the characterization (3) of proximal points, this is equivalent to

$$
\dot{u}\left(t_{1}\right)=\operatorname{prox}\left(W\left(t_{1}, u\left(t_{1}\right)\right), 0_{H}\right)
$$

i.e. analogously to what was stated in (9), $\dot{u}\left(t_{1}\right)$ equals the element of minimal norm in the ARVS.

The elements of $H$ which, similar to $\dot{u}\left(t_{1}\right)$, satisfy (14) form an affine manifold, say $W_{a}\left(t_{1}, u\left(t_{1}\right)\right.$ ) (possibly reduced to the single element $\left.\dot{u}\left(t_{1}\right)\right)$, contained in $W\left(t_{1}, u\left(t_{1}\right)\right)$. Any element of $W_{a}\left(t_{1}, u\left(t_{1}\right)\right)$ constitutes on the same footing as $\dot{u}\left(t_{1}\right)$ an apex of $W\left(t_{1}, u\left(t_{1}\right)\right)$, so the normal cone to $W\left(t_{1}, u\left(t_{1}\right)\right)$ at this point also equals $N_{C\left(t_{1}\right)}\left(u\left(t_{1}\right)\right)$. In the proximal characterization (15), $W$ may be replaced by $W_{a}$ and this makes of the determination of $\dot{u}\left(t_{1}\right)$ a linear problem.

Incidentally observe that, owing to the generation property, condition (1) implies the existence of nonpositive real numbers $\left(\lambda_{1}, \ldots, \lambda_{\kappa}\right)$ such that $\dot{u}=\Sigma_{\alpha} \lambda_{\alpha} \nabla c_{\alpha}(u)$. On account of (14), should $\alpha$ belong to $K(t, u)$ or not, in all cases one has

$$
\lambda_{\alpha} \leq 0, \quad \partial c_{\alpha} / \partial t+\dot{u} \nabla c_{\alpha} \leq 0, \quad \lambda_{\alpha}\left(\partial c_{\alpha} / \partial t+\dot{u} \nabla c_{\alpha}\right)=0
$$

a system of complementarity conditions.

\section{Approximation}

At the stage of computation, the Hilbert space $H$ naturally has finite dimension, i.e. it equals a Euclidean vector space, possibly viewed as $\boldsymbol{R}^{n}$ equipped with the standard scalar product.

\subsection{Event-driven strategy}

Such a strategy naturally applies to the situation described in Subsection 3.2. If, instead of (1), the governing equation of the process is taken in the form (15), the task becomes that of integrating an ordinary differential equation, with the difficulty that the definition of the function in the right-hand side is subject to switches. The 'events' to be monitored are the changes in the contents of the set $K(t, u(t))$. At the corresponding instants, the function $u$ remains continuous, so its value provides the initial condition from which integration should be continued over a subsequent interval. Theoretically, however, the availability of such an interval is not secured: even if the solution $u$ has been proved to exist, with high regularity (say continuous differentiability up to a certain order), the possibility remains of an accumulation of switches on the right of the primarily detected one. 


\subsection{Smoothing strategy}

Denoting by $C$ a nonempty convex subset of $H$, one shows [32] that the real function $x \mapsto(\operatorname{dist}(C, x))^{2} / 2=$ $\|x-\operatorname{prox}(C, x)\|^{2} / 2$ is Fréchet-differentiable everywhere in $H$, with gradient at point $x$ equal to $x-\operatorname{prox}(C, x)$. Choose a constant $\lambda>0$ and define the penalty function

$$
p(t, x):=\frac{\lambda}{2}(\operatorname{dist}(C(t), x))^{2} .
$$

This function takes the value 0 if the constraint $x \in C(t)$ is satisfied; otherwise its value may be interpreted as the 'fine' one has to pay for the constraint violation. If the given motion of $C$ satisfies some of the regularity assumptions we shall formulate in Section 5 below, the ordinary differential equation

$$
-\frac{\mathrm{d} u}{\mathrm{~d} t}=\nabla p(t, x)
$$

possesses a unique solution for every initial condition $u\left(t_{0}\right)=u_{0}$. It may be shown [23,35] that this solution converges to a solution of (1) when the penalty coefficient $\lambda$ tends to infinity. The differential equation (18) expresses that the velocity vector of the solution $u$ equals $\lambda$ ( $\operatorname{prox}(C, u)-u$ ). Intuitively, this tends to bring $u$ back into $C(t)$ with speed proportional to the magnitude of the violation.

\subsection{Catching-up strategy}

Devising a time-stepping scheme for the numerical approximaion of (1) involves covering the interval $I$ by a succession of adjacent subintervals. Let $\left[t_{\mathrm{i}}, t_{\mathrm{f}}\right]$ be one of them (' $\mathrm{i}$ ' as initial, ' $\mathrm{f}$ ' as final), with length $h:=t_{\mathrm{f}}-t_{\mathrm{i}}>0$. Denoting by $u_{\mathrm{i}}$ and $u_{\mathrm{f}}$ some estimates of $u\left(t_{\mathrm{i}}\right)$ and $u\left(t_{\mathrm{f}}\right)$, one approximates the time-derivative in the left-hand side of $(1)$ by $\left(u_{\mathrm{f}}-u_{\mathrm{i}}\right) / h$.

The differential inclusion (1) cannot a priori generate any Euler scheme of the explicit type: expressing that the negative of the above estimate of the derivative belongs to the normal cone at point $u_{\mathrm{i}}$ to $C_{\mathrm{i}}:=C\left(t_{\mathrm{i}}\right)$ does not allow one to calculate $u_{\mathrm{f}}$, even if the requirement that $u_{\mathrm{f}}$ should belong to $C_{\mathrm{f}}:=C\left(t_{\mathrm{f}}\right)$ is added. In contrast, the natural implicit approximation scheme merely consists in writing $u_{\mathrm{i}}-u_{\mathrm{f}} \in h N_{C_{\mathrm{f}}}\left(u_{\mathrm{f}}\right)$, where the real positive factor $h$ is immaterial, since the right-hand side is a cone. In view of (3), this is equivalent to

$$
u_{\mathrm{f}}=\operatorname{prox}\left(C_{\mathrm{f}}, u_{\mathrm{i}}\right)
$$

Starting with some initial data $u\left(t_{0}\right) \in C\left(t_{0}\right)$, the numerical procedure consists in a succession of well-defined proximations, which we call the catching-up algorithm.

The following extension is of importance when the above ideas are transported into the dynamical setting. Choose a real number $\theta>0$ and construct $t_{\mathrm{a}}:=\theta t_{\mathrm{f}}+(1-\theta) t_{\mathrm{i}}$ (where ' $\mathrm{a}$ ' stands for average), $C_{\mathrm{a}}:=C\left(t_{\mathrm{a}}\right)$, $u_{\mathrm{a}}:=\theta u_{\mathrm{f}}+(1-\theta) u_{\mathrm{i}}$. Then, $u_{\mathrm{i}}-u_{\mathrm{f}}=\left(u_{\mathrm{i}}-u_{\mathrm{a}}\right) / \theta$, so if one tries to approximate (1) by writing that an estimate of $-\mathrm{d} u / \mathrm{d} t$ should belong to $N_{C_{\mathrm{i}}}\left(u_{\mathrm{a}}\right)$, one finally obtains

$$
u_{\mathrm{f}}=\left(1-\frac{1}{\theta}\right) u_{\mathrm{i}}+\frac{1}{\theta} \operatorname{prox}\left(C_{\mathrm{a}}, u_{\mathrm{i}}\right)
$$

\section{Some existence results}

The existence of solutions to the initial value problem for the sweeping process, as formulated in Section 2 , is naturally conditioned by some regularity assumptions regarding the data $t \mapsto C(t)$. For instance, in the example presented in Subsection 3.1 of a set whose evolution is defined by applying the translation $t \mapsto a(t)$ to the fixed set $C_{0}$, the assumption of $a$ being locally absolutely continuous may be proved sufficient. Extending to more general evolutive sets the concept of absolute continuity rests on the definition of some (pseudo)distance between subsets of $H$.

Observe that a regularity assumption regarding the evolution of $C$ is needed only if, in a space-neighbourhood of the current position of $u$ and in a time-neighbourhood on the right of the current time, the set $C$ recedes. In fact, if $C$ locally expands, the sweeping process leaves $u$ unmoving. 
Generally, let $E$ be a metric space, with distance denoted by $\delta$. For two subsets $A$ and $B$ of $E$, put

$$
e(A, B):=\sup _{a \in A} \inf _{b \in B} \delta(a, b),
$$

called the metric excess of $A$ over $B$. Here, 'sup' and 'inf' are understood in the sense of the ordered set $[0,+\infty]$, entailing in particular that $e(\emptyset, B)=0$. Clearly, $e(A, B)=0$ if and only if $A$ is contained in the closure of $B$. Though $e$ does not properly constitute a distance, it may be proved that, for three subsets of $E$, the 'triangle inequality' holds [36] (attention has to be paid to $e$ lacking symmetry).

Using $e$ as it was a distance, one may introduce [36] a concept similar to that of bounded variation. Of course, no assumption of convexity for the multifunction $t \mapsto C(t)$ has place in this context. Let $[s, t]$ denote a compact subinterval of $I$. By considering all the finite sequences $\left(\tau_{i}\right)$ of the form $s=\tau_{0} \leq \cdots \leq \tau_{n}=t$ and taking the supremum of $\sum_{i=1}^{n} e\left(C\left(\tau_{i-1}\right), C\left(\tau_{i}\right)\right)$, one defines the retraction of the multifunction $C$ over the interval $[s, t]$; notation: $\operatorname{ret}(C ; s, t)$. This is zero if and only if, with respect to the ordering $C$ in the subsets of $E$, the multifunction $\tau \mapsto$ closure $C(\tau)$ is nondecreasing everywhere in $[s, t]$. If $\operatorname{ret}(C ; s, t)$ is finite for every $[s, t] \subset I$, the multifunction $C$ is said to have finite retraction over $I$. Equivalently, the nondecreasing retraction function $r: \tau \mapsto \operatorname{ret}\left(C ; t_{0}, \tau\right)$ has finite values and, whenever $s \leq t$ in $I$, one has $\operatorname{ret}(C ; s, t)=r(t)-r(s)$.

In the same context, one classically introduces the Hausdorff 'distance' between two subsets $A$ and $B$ of $E$. In the present notations, this is $h(A, B):=\max (e(A, B), e(B, A)$ ) (with values in $[0,+\infty])$. By using $h$ instead of $e$ in the above construction, one defines for every $[s, t] \subset I$ the variation of the multifunction $C$ over the interval $[s, t]$; notation: $\operatorname{var}(C ; s, t)$. This is zero if and only if the multifunction $\tau \mapsto$ closure $C(\tau)$ is constant in $[s, t]$. If the variation is finite for every $[s, t] \subset I$, the nondecreasing finite-valued variation function $v: \tau \mapsto v \operatorname{ar}\left(C ; t_{0}, \tau\right)$ yields $\operatorname{var}(C ; s, t)=v(t)-v(s)$. In any case, $\operatorname{ret}(C ; s, t) \leqslant \operatorname{var}(C ; s, t)$.

The multifunction $C$ is said to have locally absolutely continuous retraction (resp. variation) if the real function $r$ (resp. $v$ ) is absolutely continuous on every compact subinterval of $I$. More specially, the multifunction is said Lipschitz, with ratio $k$ if such is its variation function.

The above concepts are made practical by results like the following [35-38]: Let $t \mapsto A(t)$ and $t \mapsto B(t)$ be two multifunctions of a compact interval $K$ to a normed linear space $E$, with convex values. Suppose that, for every $t$ in $K$, the set $A(t)$ has nonempty intersection with the (thus nonempty) interior of $B(t)$ and that the diameter of $A(t) \cap B(t)$ is finite. Then, if both multifunctions are absolutely continuous (resp. Lipschitz), such also is the multifunction $t \mapsto A(t) \cap B(t)$.

Coming back to the sweeping process, the intuitive assumption to make in order to establish the existence of the solution to the initial value problem is that the multifunction $C$ has, locally, absolute continuous retraction. The proof is developed constructively in [38] by establishing the convergence of the catching-up algorithm. Recall also, that the approximation through penalty differential equations such as (18) is a standard method of establishing existence results for large classes of evolution problems [5]. This technique is applied to the sweeping process in [35] under the assumption that $C$ has absolutely continuous variation. Incidentally, some unusual features are displayed: by making the penalty coefficient $\lambda$ vary with $t$, the regularization approach is also used to prove the convergence of the catching-up algorithm.

\section{Weak solutions and discontinuous processes}

The catching-up procedure of Section 4 consists in constructing inductively a sequence of points through successive proximations into a sequence of sets, without reference to the corresponding values of $t$. Going to the limit when discretization is refined, one infers that the sweeping process itself should associate the successive positions of the moving point $u$ to the successive positions of the moving set $C$ in a way which does not depend on the timing. In fact, let $\pi: t^{\prime} \mapsto t$ be a nondecreasing locally absolutely continuous map of some interval $I^{\prime}$ onto $I$. The derivative of $u^{\prime}: t^{\prime} \mapsto u\left(\pi\left(t^{\prime}\right)\right)$ is expressed through the chain rule as $(\mathrm{d} u / \mathrm{d} t)\left(\mathrm{d} t / \mathrm{d} t^{\prime}\right)$, with $\mathrm{d} t / \mathrm{d} t^{\prime} \geqslant 0$. Because the right-hand side of (1) is a cone, one sees that $u^{\prime}$ is a solution of the sweeping process on the time-interval $I^{\prime}$ by the moving set $t^{\prime} \mapsto C\left(\pi\left(t^{\prime}\right)\right)$. Generally, in mechanics, governing relations which enjoy a property of this sort are qualified as rate-indifferent.

The convergence proof of the catching-up algorithm (more precisely, the convergence of a net of stepfunctions similarly constructed) works under the mere assumption that the moving set $C$ has locally bounded 
retraction [38] (a fortiori if it has locally bounded variation) and its retraction over every subinterval of $I$ is found to majorize the corresponding variation of the limit function $u$. The latter may be called a weak solution to the sweeping process. The following explains how this generalization remains connected with the primary formulation (1).

Recall that, with a function $u: I \rightarrow H$ of locally bounded variation, an $H$-valued measure on $I$ is associated, called its differential measure (or Stieltjes measure), that we shall denote by $\mathrm{d} u$. The function $u$ is locally absolutely continuous if and only if this vector measure admits a density function relative to the Lebesgue measure in $I$, which is nothing but $\dot{u}[13,44]$. If one denotes by $\mathrm{d} t$ the Lebesgue measure in $I$ (it in fact equals the differential measure of the real function $t \mapsto t$ ), the symbol $\mathrm{d} u / \mathrm{d} t$ in (1) may be read as the density function of the vector measure $\mathrm{d} u$ relatively to the positive real measure $\mathrm{d} t$.

Suppose now that $u$ is a weak solution. Since it has locally bounded variation, some non-negative real measures on $I$ are known to exist, among them the differential measure $\mathrm{d} r$ of the retraction function of $C$, relatively to which $\mathrm{d} u$ admits density functions. Restricting oneself, for simplicity, to the case where the retraction function $r$ is right-continuous, one establishes [27,38] that the density function $\mathrm{d} u / \mathrm{d} r$ verifies the same inclusion as in (1) with the possible exception of a $\mathrm{d} r$-negligible subset of $I$. Because the right-hand side is a cone, this inclusion is preserved if one replaces $\mathrm{d} r$ by any non-negative real measure on $I$ relatively to which $\mathrm{d} u$ possesses a density function: this reflects, in the present context, the rate-indifference property mentioned in the foregoing. For more precision about such changes of 'base' measures and the technical handling of possible negligible sets, one may refer to [44]. It is thus natural to avoid mentioning any specific base measure and to formulate the sweeping process in the form

$$
-\mathrm{d} u \in N_{C(t)}(u(t))
$$

called a measure differential inclusion. Governing conditions of this sort play a significant role in 'nonsmooth dynamics'.

Observe that, if the right-continuous retraction function $r$ is discontinuous, a weak solution $u$ (it is also right-continuous) may exhibit jumps. If this occurs at some point $t_{1}$ of $I$, the vector measure $\mathrm{d} u$ possesses at this point an atom with mass $u\left(t_{1}\right)-u^{-}\left(t_{1}\right)$. Through the characterization (23) of proximal points, one derives the jump law $u\left(t_{1}\right)=\operatorname{prox}\left(C\left(t_{1}\right), u^{-}\left(t_{1}\right)\right)$.

Concerning the initial value problem for weak, possibly discontinuous, solutions of the sweeping process, uniqueness rests on the same non-expansion property as in Subsection 1, but instead of the elementary differentiation of the real function $t \mapsto\left\|u_{2}(t)-u_{1}(t)\right\|^{2}$, one has to base the proof on the Calculus of differential measures. What follows is established in [44] as a special case of some rules of this Calculus.

Let $u: I \rightarrow H$ have locally bounded variation. Then, the $H$-valued functions $t \mapsto u^{-}(t)$ and $t \mapsto u^{+}(t)$ (the leftand right-limits which, classically, are sure to exist) have locally bounded variation, as well as the real function $t \mapsto\|u(t)\|^{2}$ and the following inequalities hold in the sense of the ordering of real measures on $I$

$$
u^{-} \cdot \mathrm{d} u \leqslant \frac{1}{2} \mathrm{~d}\|u\|^{2} \leqslant u^{+} . \mathrm{d} u .
$$

Concerning the approximation of weak solutions, a useful tool for assessing the discrepancy between two, possibly discontinuous, functions is the Hausdorff distance of their graphs (or of certain completed sets), as subsets of the metric space $I \times H[28,39]$.

\section{Going to dynamics}

\subsection{Parametrization}

Let the possible configurations of a mechanical system be parametrized through generalized coordinates, say $q:=\left(q^{1}, q^{2}, \ldots, q^{n}\right)$. This, at least, holds locally: the parametrization of the whole set of the possible configurations commonly requires the introduction of several overlapping charts; when each of these charts is used, the element $q$ has only to range in an open subset of $\boldsymbol{R}^{n}$. For brevity, no further allusion will be made to this.

The construction of such a parametrization is a standard step in the dynamics of rigid body collections, 
possibly by taking profit of the presence of frictionless linkages, in order to make the number $n$ as small as possible. This being done, one takes here into account the constraints of non-interpenetrability whose geometric effect is assumed expressed by a finite set of inequalities

$$
f_{\alpha}(t, q) \leqslant 0, \quad \alpha \in\{1,2, \ldots, \kappa\},
$$

where $f_{1}, f_{2}, \ldots, f_{\kappa}$ are given functions. Equality $f_{\alpha}=0$ corresponds to the occurrence of a contact. Through the presence of $t$ as an argument of $f_{\alpha}$, provision is made for the inequality to describe the confinement of a part of the system by some external boundary with prescribed motion.

As an example, one may consider a pair of members of the system whose positions in a chosen reference frame are well located as soon as the value of the element $q$ of $\boldsymbol{R}^{n}$ is known (together with the time $t$ in case of a 'rheonomic', i.e. time-dependent, parametrization). Then, one may take as $f_{\alpha}$ the expression, as a function of $(t, q)$, of some measure of the overlap of the two bodies. This overlap should be understood as a directed quantity, so that it becomes negative in case the bodies lie apart from each other. The convention applied in (23), of characterizing the permitted configurations through the $\leqslant 0$ inequality, comes from convex optimization theory, where such a sign convention offers technical advantages. No convexity hypothesis is made here concerning the functions $f_{\alpha}$ : since such an assumption would not be preserved under a change of parametrization, it cannot, in general, have any mechanical meaning (note, however, that the assumption of a convex feasible set in $\boldsymbol{R}^{n}$ proves mathematically fruitful in [49]). If one prefers to deal with the $\geqslant 0$ symbol, there is only to consider, instead of the overlap the opposite quantity, usually called the gap between the considered bodies.

The above formalism is not limited to collections of strictly rigid bodies, since $q$ may also include parameters accounting for a finite-freedom approximation of deformability. Such additional parameters possibly arise from some modal representation of the deformation dynamics or from the Finite Element discretization of deformable parts (see e.g. the contribution of M. Jean in this volume).

The geometric characterization of non-interpenetration through a finite set of inequalities such as (23) is operative in most practical situations. For instance, it is still valid in the case of a body touching the smooth surface of another one by a sharply pointed asperity. In contrast, non-interpenetration cannot be described anymore in that way in the neighbourhood of a configuration where two sharp asperities come into contact by their points. In practice, algorithms based on (23) are found to still perform acceptably in such irregular circumstances.

In all the sequel, it will be assumed that each function $f_{\alpha}$ is $\mathscr{C}^{1}$, with $\partial f_{\alpha} / \partial q \neq 0$ at least in a neighbourhood of the hypersurface $f_{\alpha}=0$ of $\boldsymbol{R}^{n+1}$.

For every imagined motion $t \mapsto q(t)$ and for $t$ such that the derivative $u:=\mathrm{d} q / \mathrm{d} t$ exists, the kinetic energy has a generic expression of degree 2 in $u$, say

$$
\mathscr{E}_{k}(t, q, u)=\frac{1}{2} A_{i j}(t, q) u^{i} u^{j}+B_{i}(t, q) u^{i}+C(t, q)
$$

where $A$ is a symmetric positive definite $n \times n$-matrix, $B \in \boldsymbol{R}^{n}$ and $C \in \boldsymbol{R}$. In the common case of a 'scleronomic' (i.e. time-independent) parametrization, $A$ is constant in $t$, while $B$ and $C$ vanish.

The equations of dynamics, to be invoked in the sequel, involve both unknown functions $t \mapsto q$ and $t \mapsto u$. We have just introduced the latter as the derivative of the former. Actually, in existential studies as well as in the $\mathrm{CD}$ approximation technique, the emphasis is primarily placed on the velocity function $t \mapsto u$, to which the position function $t \mapsto q$ shall be related through

$$
q(t)=q\left(t_{0}\right)+\int_{t_{0}}^{t} u(s) \mathrm{d} s .
$$

Practically, it is sometimes advantageous to describe the velocity state of the system by a velocity function connected with $q$ in a more complicated way. For instance, when dealing with 3-dimensional rigid bodies, it is usual to attach to each of them a frame of principal axes of inertia emanating from its center of mass. Then, one may choose to enter, as members of the $\boldsymbol{R}^{n}$-valued function $u$, the components relative to these axes of the spin vector of the rigid body, instead of the time-derivatives of some directional parameters. This brings considerable advantage of generating a contribution in the matrix $A$ which is diagonal and constant with regard to $t$ and $q$. Retrieving from the spin components the evolution of the directional parameters of the rigid body is only a 
matter of integrating some standard kinematical formulas. Incidentally, observe that in the dynamics of a collection of $k$ isotropic spherical beads, a popular model of granular system, the proper orientation of each bead is immaterial: only its spin vector counts. Hence, if the above procedure is applied, computation involves a velocity function with $6 k$ components while the position function has only $3 k$ components.

\subsection{Contact kinematics}

In order to enter into the equations of dynamics the available phenomenological information concerning the contact interactions, one has to connect the local description of contact in physical space with the parametrization introduced above.

Suppose that inequality $f_{\alpha} \leqslant 0$ expresses the non-interpenetration of some pair of members of the system, say $\mathscr{B}$ and $\mathscr{B}^{\prime}$, so that equality $f_{\alpha}=0$ corresponds to these bodies touching each other at some point of space denoted by $M_{\alpha}$. We shall assume this to be an isolated contact point, but other contacts, associated with different values of $\alpha$, may also be in effect between the same bodies at the same instant. For every imagined motion $t \mapsto q(t)$ bringing the system through the considered contacting position for some value of $t$, the velocity vectors $\mathscr{V}_{\alpha}$ and $\mathscr{V}_{\alpha}^{\prime}$ of the respective particles of $\mathscr{B}$ and $\mathscr{B}^{\prime}$ passing at point $M_{\alpha}$ let themselves be expressed as affine functions of the value $u \in R^{n}$ of the velocity function. The same is thus true for the relative velocity $\mathcal{U}_{\alpha}=\mathscr{V}_{\alpha}-\mathscr{V}_{\alpha}^{\prime}$ of $\mathscr{B}$ with respect to $\mathscr{B}^{\prime}$ at this point, say

$$
\mathcal{U}_{\alpha}=G_{\alpha} u+\mathscr{W}_{\alpha},
$$

where $G_{\alpha}: \boldsymbol{R}^{n} \rightarrow \boldsymbol{R}^{3}$ denotes a linear mapping, depending on $t$ and $q$. No attention is paid at this stage to the imagined motion preserving contact or not. The term $\mathscr{W}_{\alpha} \in \boldsymbol{R}^{3}$, a known function of $t$ and $q$, vanishes in the familiar case of a scleronomic parametrization.

Assume that the contact actions that body $\mathscr{B}$ experiences at point $M_{\alpha}$ from body $\mathscr{B}{ }^{\prime}$ are described as a simple force $\mathscr{R}^{\alpha}$ (there would be no conceptual difficulty in adding to this description some local torque, accounting for a resistance to rolling). Then $\mathscr{B}^{\prime}$ experiences from $\mathscr{B}$ the force $-\mathscr{R}^{\alpha}$. The standard machinery of analytical dynamics needs a representation of this pair of forces, in regard to the chosen parametrization, through its covariant components (or 'generalized components'), namely the element $r^{\alpha}$ of $\boldsymbol{R}^{n}$ expressed as

$$
r^{\alpha}=G_{\alpha}^{*} \mathscr{R}^{\alpha}
$$

with $G_{\alpha}^{*}: \boldsymbol{R}^{3} \rightarrow \boldsymbol{R}^{n}$ denoting the transpose of $G_{\alpha}$.

The convention of implicit summation will never be applied to Greek indices.

Similar formulas are found to hold if inequality $f_{\alpha} \leqslant 0$ expresses the confinement of a part $\mathscr{B}$ of the system by some external boundary with prescribed motion. Assume that equality $f_{\alpha}=0$ corresponds to contact taking place at some point, here again denoted by $M_{\alpha}$. The local velocity, at this point, of the body $\mathscr{B}$ with respect to the boundary has the same form as $\mathscr{U}_{\alpha}$ in (26), where $\mathscr{W}_{\alpha}$ now takes into account the known velocity of the boundary. This makes $r^{\alpha}$ in (27) consist of the covariant components of the force $\mathscr{R}^{\alpha}$ alone, acting on $\mathscr{B}$. Its counterpart exerted by $\mathscr{B}$ upon the boundary is not a force experienced by the system.

In both cases, the following relationship is found to hold [43] between the element $\partial f_{\alpha} / \partial q$ of $\boldsymbol{R}^{n}$ and the normal unit vector $\boldsymbol{n}^{\alpha}$ at point $M_{\alpha}$ to the contacting bodies, directed toward $\mathscr{B}$

$$
\exists \lambda_{\alpha} \geqslant 0 \quad \text { such that } G_{\alpha}^{*} n^{\alpha}=-\lambda_{\alpha} \frac{\partial f_{\alpha}}{\partial q} .
$$

The proof of this rests on a 'unilateral' version of the algebraic theorem of Lagrange multipliers, known in Convex Analysis as Farkas' lemma [51].

In all the sequel, we shall assume that the mapping $G_{\alpha}$ is surjective of $\boldsymbol{R}^{n}$ onto $\boldsymbol{R}^{3}$; equivalently, its transpose $G_{\alpha}^{*}$ is injective of $\boldsymbol{R}^{3}$ into $\boldsymbol{R}^{n}$. Then, $\lambda_{\alpha}$ in (28) is nonzero. Some special positions of certain linkages may give rise to 'wedging' effects which contradict this assumption.

\subsection{The equations of Dynamics}

The expression (24) of the kinetic energy allows one to write down the dynamical equations of the system by using the Lagrange technique, possibly adapted in order to accept, as explained in Subsection 7.1, the handling of velocity parameters not equal to the time-derivatives of position parameters, 


$$
A(t, q) \frac{\mathrm{d} u}{\mathrm{~d} t}=F(t, q, u)+\sum_{\alpha} r^{\alpha}
$$

an equality of elements of $\boldsymbol{R}^{n}$. The expression $F$ comprises standard terms of the Lagrange equations and the covariant components of some applied forces, supposed given as functions of the time, the position of the system and its velocity. The elements $r^{\alpha}, \alpha \in\{1,2, \ldots, \kappa\}$, are the covariant components of the contact forces as defined in (27): $r^{\alpha}$ can be nonzero only when inequality $f_{\alpha}(t, q) \leqslant 0$ holds as an equality.

To the above equation, the geometric conditions (23) of non-interpenetration are to be adjoined and also some phenomenological information concerning each possible contact, called a contact law, for which we assume the form

$$
\operatorname{law}_{\alpha}\left(t, q, \mathscr{U}_{\alpha}, \mathscr{R}^{\alpha}\right)=\text { true. }
$$

The local relative velocity $\mathcal{U}_{\alpha}$ and the contact force $\mathscr{R}^{\alpha}$ are respectively connected with $u$ and $r^{\alpha}$ through (26) and (27).

The simplest example of such a law is provided by frictionless contact.

\section{Frictionless contact dynamics}

\subsection{Basic formulation}

Traditional mechanics grants precedence to the special case of ideal i.e. frictionless constraints. In the present setting, this means that, at the contact point denoted in Subsection 7.2 by $M_{\alpha}$, the contact force $\mathscr{R}^{\alpha}$ has the direction of the normal vector $\boldsymbol{n}^{\alpha}$. In addition, it is assumed that the contact exhibits no cohesion (gluing) effect, hence $\exists \rho_{\alpha} \geqslant 0: \mathscr{R}^{\alpha}=\rho_{\alpha} n^{\alpha}$. In view of (28), this is equivalent to

$$
\exists \mu_{\alpha} \leqslant 0 \quad \text { such that } \quad r^{\alpha}=\mu_{\alpha} \frac{\partial f_{\alpha}}{\partial q} .
$$

Here, it is implicitely assumed that the contact with label $\alpha$ is effective, i.e. the constraint inequality $f_{\alpha}(t, q) \leqslant 0$ holds as equality. Otherwise, the contact force is essentially zero, so one may understand that $\mu_{\alpha}=0$ if $f_{\alpha}<0$. As such an alternative is offered for every $\alpha=1, \ldots, \kappa$, one recognizes once more the familiar pattern of a system of complementarity conditions. In the context of formulating contact laws, these are commonly referred to as Signorini's conditions [52]. What follows amounts to developing the same in different notations.

Let $\Phi(t)$ denote the subset of $\boldsymbol{R}^{n}$ consisting of the positions $q$ agreeing at time $t$ with the non-interpenetration inequalites (23), called the feasible region at time $t$. For every $t$ and $q$, put

$$
J(t, q):=\left\{\alpha \in\{1, \ldots, \kappa\} \mid f_{\alpha}(t, q) \geqslant 0\right\} .
$$

If $q \in \Phi(t)$, this equals the set of the values of $\alpha$ such that (23) holds as an equality, i.e. the subset of $\{1, \ldots, \kappa\}$ corresponding to effective contacts. But, in existential studies, as well as in numerical computation, it proves useful to apply the definition (32) also to positions implying some violation of the non-interpenetration inequalities.

Concerning the elements $\boldsymbol{n}^{\alpha}$ and $G_{c r}$ introduced in Subsection 7.2, one may similarly agree to extend their definitions (in a smooth way) to values of $q$ not belonging to $\Phi(t)$, i.e. to define them at least on a neighbourood of the hypersurface $f_{\alpha}=0$. This extension may even be constructed in a way which preserves (28).

When $q$ in $\Phi(t)$ belongs to the hypersurface $f_{\alpha}=0$, i.e. when $\alpha \in J(t, q)$, the $n$-vector $\partial f_{\alpha} / \partial q$ (we have assumed it to be nonzero) classically defines the normal direction in $\boldsymbol{R}^{\prime \prime}$ to this hypersurface at point $q$, directed outward of the region $\Phi(t)$. The situation differs from what we have met in Subsection 3.2 by that no convexity concept applies to the functions $f_{\alpha}$ nor to the region $\Phi$. In the convex analytic definition of the normal cone recalled in Subsection 2.1, the convex set $C$ was involved globally. But in the manifold of the system positions only local operations make sense, so we shall resort to a different conception of a so-called normal cone. In contrast, the convex analytic standpoint prevails when we work in the space of velocities, which is a linear space. 
Let us agree to denote by $N_{\Phi(t)}(q)$ the convex cone generated in $\boldsymbol{R}^{n}$ by the vectors $\partial f_{\alpha} / \partial q, \alpha \in J(t, q)$ and call it the (outward) normal cone to the region $\Phi(t)$ at the point $q$ of its boundary. If $q$ lies in the interior of $\Phi(t)$ the set $J(t, q)$ is empty so, according to standard conventions, the generated cone reduces to the zero of $\boldsymbol{R}^{\mathrm{n}}$.

When evaluating the term $\Sigma_{\alpha} r^{\alpha}$ in the dynamical equation (29), it is enough to make $\alpha$ range in $J(t, q)$ since otherwise $\mathscr{R}^{\alpha}$ vanishes. Thus, in view of (31), the negative of the sum $\Sigma_{\alpha} r^{\alpha}$ belongs to $N_{\Phi(t)}(q)$. Conversely, every element of $\boldsymbol{R}^{n}$ whose negative belongs to this cone admits a decomposition into the sum of the covariant components of forces $\mathscr{R}^{\alpha}$ which are feasible as reactions of the corresponding frictionless contacts. So, the traditional goal of analytical dynamics, namely the elimination of the unknown reactions of ideal constraints, is attained by rewriting the dynamical equation (33) in the form of the differential inclusion

$$
F(t, q, u)-A(t, q) \frac{\mathrm{d} u}{\mathrm{~d} t} \in N_{\Phi(t)}(q)
$$

to which the non-interpenetration inequalities (23) have to be adjoined. A notational trick allowing one to incorporate the latter in the writing (33) is to agree that $N_{\Phi(t)}(q)$ is empty when $q \notin \Phi(t)$.

$R E M A R K$. Since in defining $N_{\Phi(t)}(q)$, we depart from the convex analytic standpoint presented in Section 2 , the connection of this cone with the possible motion of points in the region $\Phi(t)$ is left to be discussed. At a fixed time $t$, let us consider a chain of points $\tau \mapsto \tilde{q}(\tau) \in \Phi(t)$ with $\tilde{q}(0)=q$ (in traditional analytical dynamics, this is called a virtual motion at time $t$ ). A simplified variant of the reasoning developed in the next Subsection shows that the right-derivative of $\tilde{q}$ at $\tau=0$, if it exists, necessarily belongs to the polar cone $\left(N_{\Phi(t)}(q)\right)^{*}$ in the sense of the standard scalar product of $\boldsymbol{R}^{n}$. It is natural to call this the tangent cone to the region $\Phi(t)$ at point $q$. However, in the absence of additional assumption (the usual one is that $\left(N_{\Phi(t)}(q)\right)^{*}$ have nonempty interior, a fact equivalent to $N_{\Phi(t)}(q)$ possessing a bounded section) an element of this so-called tangent cone does not necessarily correspond to a chain of points in $\Phi(t)$ as considered above (see [41] for a counter-example and [1] for the sufficiency of additional assumptions). Anyway the investigation of variants in the definition of normal or tangent cones to a point set is a vast subject (see e.g. [7,19]).

\subsection{Handling non-interpenetration in terms of velocities}

The differential inclusion (33) is found uneasy to approximate. The move we are to make now is crucial in the elaboration of the contact dynamics method.

Let us consider, at time $t_{1}$, a position $q_{1}$ such that the contact with label $\alpha$ is in effect, i.e. $f_{\alpha}\left(t_{1}, q_{1}\right)=0$. If a motion $t \mapsto q(t)$, starting from this position, complies with the non-interpenetration inequality $f_{\alpha} \leqslant 0$ for every $t \geqslant t_{1}$, the right-derivative at point $t_{1}$ of the real function $t \mapsto f_{\alpha}(t, q(t))$ necessarily is $\leqslant 0$. Since $f_{\alpha}$ has been supposed $\mathscr{C}^{1}$, this right-derivative exists as soon as the function $t \mapsto q(t)$ possesses a right-derivative at point $t_{1}$, say $u^{+}$, and is expressed through the chain rule in the form $\partial f_{\alpha} / \partial t+u^{+} \partial f_{\alpha} / \partial q$. Therefore, if a motion satisfies the non-interpenetration inequalities, the right-velocity $u^{+}(t)$, whenever it exists, belongs to the polyhedral closed convex set

$$
W(t, q):=\left\{v \in \boldsymbol{R}^{n} \mid \forall \alpha \in J(t, q): \frac{\partial f_{\alpha}}{\partial t}+v \frac{\partial f_{\alpha}}{\partial q} \leqslant 0\right\} .
$$

Symmetrically, the left-velocity $u^{-}(t)$, whenever it exists, belongs to the set $W^{\prime}(t, q)$ defined by reversing the inequalities.

The situation here is similar to that we met in Subsection 3.2. Let the considered motion satisfy the differential inclusion (33) in the conventional sense, i.e. $t \mapsto q$ is locally absolutely continuous and $u(t)$ equals for almost every $t$ its (bilateral) derivative. Then, what precedes shows that, for such $t$, the value of $u$ belongs the intersection $W^{\prime} \cap W$, hence

$$
\forall \alpha \in J(t, q): \quad \frac{\partial f_{\alpha}}{\partial t}+u \frac{\partial f_{\alpha}}{\partial q}=0 .
$$

By substraction one obtains that $v$ belongs to $W(t, q)$ if and only if the inequality $(v-u) \partial f_{\alpha} / \partial q \leqslant 0$ holds for every $\alpha$ in $J(t, q)$. In other words, $W(t, q)=u+\left(N_{\Phi(t)}(q)\right)^{*}$. Since the normal cones at corresponding points of 
translate sets are equal, there comes out that $N_{W(t, q)}(u)$ equals the normal cone to $\left(N_{\Phi(t)}(q)\right)^{*}$ at the origin, namely $N_{\Phi(t)}(q)$ in view of (6). Consequently, if the function $u$ satisfies (33), it also satisfies the differential inclusion

$$
F(t, q, u)-A(t, q) \frac{\mathrm{d} u}{\mathrm{~d} t} \in N_{W(t, q)}(u) .
$$

This makes a priori a stronger requirement than (33), since $N_{W(t, q)}(u)$ is essentially a subset of $N_{\Phi(t)}(q)$.

This inclusion implies that $u \in W(t, q)$; otherwise, the right-hand side would be empty. It is thus useful to observe [43]:

THE INTEGRATION LEMMA. Let $q: I \rightarrow \boldsymbol{R}^{n}$ be connected with a locally Lebesgue-integrable function $u: I \rightarrow \boldsymbol{R}^{n}$ through the integration formula (25). Suppose that $q\left(t_{0}\right) \in \Phi\left(t_{0}\right)$ and that $u(t) \in W(t, q)$ for almost every $t$ in $I$. Then, $q(t) \in \Phi(t)$ for every $t$ in $I$.

REMARK. For brevity, it has been implicitely assumed in what precedes that $u$ equals the time-derivative of $q$. That the same interplay of inequalities works, more generally, when other sorts of velocity parameters are used could be established through the machinery of Subsection 7.2, by taking for instance as function $f_{\alpha}$ the normal overlap of the concerned bodies (negative if the bodies lie apart). By elementary kinematics, one finds that, for an arbitrary motion, the time-derivative of this overlap equals $-n^{\alpha} . \mathscr{U}_{\alpha}=-n^{\alpha} . G_{\alpha} u-n^{\alpha} . \mathscr{W}$, expressions of the same form as those in (34) and (35).

\subsection{A primitive example of $C D$ algorithm}

Let some initial conditions $q\left(t_{0}\right)=q_{0} \in \Phi\left(t_{0}\right)$ and $u\left(t_{0}\right)=u_{0} \in W\left(t_{0}, q_{0}\right)$ be imposed on a solution to the differential inclusion (36). We propose here a time-stepping procedure for the approximation of the functions $u$ and $q$. As in Section 4, let $\left[t_{\mathrm{i}}, t_{\mathrm{f}}\right]$ denote a time-step, with length $h$. From the computed values $u_{\mathrm{i}}$ and $q_{\mathrm{i}}$ of the functions $u$ and $q$ at $t=t_{\mathrm{i}}$, which result from the preceding step, one has to predict $u_{\mathrm{f}}$ and $q_{\mathrm{f}}$ corresponding to $t_{\mathrm{f}}$.

The given function $F$ and $A$ in (36) usually depend smoothly on their arguments, so we approximate their values throughout the concerned interval by fixing $t$ as the 'midtime' $t_{\mathrm{m}}:=t_{\mathrm{i}}+h / 2$ and $q$ as the 'midposition' $q_{\mathrm{m}}:=q_{\mathrm{i}}+h u_{\mathrm{i}} / 2$ while $u$ is made equal to $u_{\mathrm{i}}$.

Similarly, the detection of the contacts to be treated as effective in the time-step is based on the values that the functions $f_{\alpha}$ take at point $\left(t_{\mathrm{m}}, q_{\mathrm{m}}\right)$, i.e. the set of the 'active' values of $\alpha$ is estimated to be $J_{\mathrm{m}}:=J\left(t_{\mathrm{m}}, q_{\mathrm{m}}\right)$ and $W(t, q)$ to equal the constant subset $W_{\mathrm{m}}:=W\left(t_{\mathrm{m}}, q_{\mathrm{m}}\right)$ of $\mathrm{R}^{n}$.

The differential inclusion (36) is formally analogous to that of the sweeping process by the moving set $W$ hence, for the same reason as in Section 4, we choose to evaluate the unknown $u_{\mathrm{f}}$ through a procedure of the implicit type. Approximating as usual $\mathrm{d} u / \mathrm{d} t$ by $\left(u_{\mathrm{f}}-u_{\mathrm{i}}\right) / h$, this leads to

$$
h F\left(t_{\mathrm{m}}, q_{\mathrm{m}}, u_{\mathrm{i}}\right)-A\left(t_{\mathrm{m}}, q_{\mathrm{m}}\right)\left(u_{\mathrm{f}}-u_{\mathrm{i}}\right) \in N_{w_{\mathrm{m}}}\left(u_{\mathrm{f}}\right)
$$

(the right-hand side being a cone, the multiplier $h>0$ has been dropped).

This characterizes the unknown $u_{\mathrm{f}}$ as the solution of a quadratic programming problem. One achieves the same simplicity of notation as in Section 4 by taking for $A$ the identity matrix (this actually entails no loss of generality, but amounts to equip the linear space where $u$ takes its values with the 'kinetic' Euclidean metric; some precisions on the implementation of this trick are given in [41]). In view of (3), the inclusion (37) is then found equivalent to

$$
u_{\mathrm{f}}=\operatorname{prox}\left(W_{\mathrm{m}}, u_{\mathrm{i}}+h F_{\mathrm{m}}\right) \text {. }
$$

Finally, one completes the computation step with

$$
q_{\mathrm{f}}=q_{\mathrm{m}}+\frac{h}{2} u_{\mathrm{f}}
$$

REMARK 1. The calculation of $u_{\mathrm{f}}$ from $u_{\mathrm{i}}$ is based on mechanical elements evaluated at the mid-position $q_{\mathrm{m}}$; in turn, $u_{\mathrm{f}}$ is used to calculate from $q_{\mathrm{m}}$ the final position of the current step and, from there, the mid-position of the 
subsequent time-step. This interleaving makes the above time-stepping procedure resemble the policy called 'leapfrog' in molecular dynamics simulations. In order to compare its precision with that of an ordinary Euler explicit time-stepping scheme, one may apply it to calculating the parabolic unconstrained motion of a particle in a uniform gravity field. If $h$ is constant, the values found for $q$ at the successive steps coincide with the exact solution, while the Euler scheme generates cumulative errors. Of course, when an algorithm of the above sort is implemented with constant $h$, one may calculate each $q_{\mathrm{m}}$ from the preceding one by a single incrementation. The proper output of the computation, namely $q_{\mathrm{f}}$, may not be needed at each step.

REMARK 2. Since the differential inclusion (36) is invoked as governing the evolution, one relies on the 'integration lemma' of Subsection (8.2) in expecting that the computed solution shall agree with the non-interpenetration inequalities. The current practice in the numerical dynamics of machines analogously rests on the imposition of velocity conditions in order to enforce some constraint equalities. It is commonly observed in this case that the discretization errors, inherent in time-stepping methods, generate cumulative discrepancies, so that one has to resort to some auxiliary techniques of 'constraint stabilization'. From our experiments with the CD handling of the non-interpenetration inequalities, we may say that, provided the step length remains below some critical level, $\mathrm{CD}$ algorithms are free from this defect, due to a certain self-correction connected with the feasible region $\Phi$ possessing a nonempty interior and having curved boundaries.

\subsection{Handling non-interpenetration in terms of accelerations}

The above time-stepping scheme of the implicit type, rests on predicting the velocity $u$ whithout resorting to any expression of the acceleration $\dot{u}$. In contrast, what follows is aimed at determining the right-acceleration $\dot{u}^{+}$, which is needed when a time-stepping scheme of the explicit type is implemented. This is usually what one does when the 'event driven strategy' is adopted.

Let us assume for this Subsection that the functions $f_{\alpha}$ are $\mathscr{C}^{2}$ in the concerned region of $\boldsymbol{R}^{n+1}$. Let $t_{1}$ denote an instant preceded by some time-interval throughout which the motion satisfies (33). This hypothesis involves that $t \mapsto u(t)$ is absolutely continuous on this interval, so (35) is verified, expressing that the function $t \mapsto f_{\alpha}(t, q(t))$ has zero derivative of order one. Assume that $u$ remains continuous also at instant $t_{1}$, i.e. no collision occurs.

The investigation of the subsequent motion through an event-driven policy rests on the assumption that $t_{1}$ is followed by a nonzero interval throughout which (33) is verified again. It only may happen that some contacts break at $t_{1}$, inducing a change of $J(t, q)$. If $u$ possesses a derivative on the right of $t_{1}$, say $\dot{u}_{1}^{+}$, the function $t \mapsto f_{\alpha}(t, q(t))$ possesses a second derivative on the right of $t_{1}$, expressed through the chain rule in the form $a_{\alpha}\left(t_{1}, q_{1}, u_{1}\right)+\dot{u}_{1}^{+} \partial f_{\alpha} / \partial q$. Since the function is zero at $t_{1}$, as well as its first derivative, non-interpenetration requires of this second derivative to be $\leqslant 0$. If it is strictly negative, $f_{\alpha}(t, q(t))$ becomes strictly negative on a subsequent interval, making the corresponding quantities $r^{\alpha}=\mu_{\alpha} \partial f_{\alpha} / \partial q$ vanish on this interval, as well as their limits for $t \downarrow t_{1}$, assumed to exist. One thus obtains a set of complementarity conditions

$$
a_{\alpha}+\dot{u}_{1}^{+} \partial f_{\alpha} / \partial q \leqslant 0, \quad \mu_{\alpha} \leqslant 0, \quad\left(a_{\alpha}+\dot{u}_{1}^{+} \partial f_{\alpha} / \partial q\right) \mu_{\alpha}=0 .
$$

By joining them to the equation of Dynamics

$$
A \dot{u}_{1}^{+}-F=\sum_{\alpha} \mu_{\alpha} \partial f_{\alpha} / \partial q
$$

one reduces the determination of $\dot{u}_{1}^{+}$and of the multipliers $\mu_{\alpha}$ to a linear complementarity problem in standard form.

Because the matrix $A$ is positive definite, such a problem is classically equivalent to minimizing a convex quadratic function in a closed convex polyhedral subset of $\boldsymbol{R}^{n}$. It has been shown in $[31,33]$ that this extremal characterization of the acceleration may be viewed as extending Gauss' principle of the least deviation to mechanical systems subject to unilateral frictionless constraints. Some dual minimization property characterizes the contact forces. 


\section{Back to contact locus}

\subsection{Complete contact laws}

Let us consider the contact with label $\alpha$. In the notations of Section 7.2, put

$$
\mathscr{K}_{\alpha}(t, q):= \begin{cases}\left\{\mathscr{V} \in \boldsymbol{R}^{3} \mid \mathscr{V} . n^{\alpha} \geqslant 0\right\} & \text { if } \mathrm{f}_{\alpha}(t, q) \geqslant 0 \\ \boldsymbol{R}^{3} & \text { otherwise. }\end{cases}
$$

This closed convex subset of $\boldsymbol{R}^{3}$ constitutes the set of the values of the local right-velocity of $\mathscr{B}$ relatively to $\mathscr{B}^{\prime}$ (the latter may be an external boundary) which at the considered instant are compatible with non-interpenetration, namely the ARVS in the terminology of Section 3. In the first line of above definition, $\mathscr{K}_{\alpha}$ equals a half-space, so the normal cone $N_{\mathscr{K}_{\alpha}}$, in the convex analytic sense, evaluated at the origin $\mathbf{0}$ of $\boldsymbol{R}^{3}$, equals the half-line generated by $-\boldsymbol{n}^{\alpha}$. In case $\mathscr{K}_{\alpha}=\boldsymbol{R}^{3}$, the cone $N_{\mathscr{K}_{\alpha}}(\boldsymbol{0})$ reduces to the set $\{\boldsymbol{0}\}$.

Therefore, the no-friction and no-gluing assumptions, as formulated in Subsection 8.1 (including the case of no-contact, since it has been agreed to keep the local elements $n^{\alpha}, G_{\alpha}$, etc. meaningful in this case) are equivalent to assert

$$
-\mathscr{R}^{\alpha} \in N_{\mathscr{K}_{\alpha}^{(t, q)}}(\mathbf{0}) \text {. }
$$

The move made in Subsection 8.2 of replacing inclusion (33) by inclusion (36) amounts in the present context to replace inclusion (39) by

$$
-\mathscr{R}^{\alpha} \in N_{\mathscr{H}_{\alpha}(t, q)}\left(\mathscr{U}_{\alpha}\right) \text {, }
$$

with $\mathcal{U}_{\alpha}$ denoting as before the velocity of $\mathscr{B}$ relative to $\mathscr{B}^{\prime}$ at point $M_{\alpha}$. In fact, during an episod of persistent contact, $\mathscr{U}_{\alpha}$ belongs to the boundary plane of $\mathscr{K}_{\alpha}$, so $N_{\mathscr{H}_{\alpha}}\left(\mathscr{U}_{\alpha}\right)=N_{\mathscr{K}_{\alpha}}(\mathbf{0})$, while in case of no-contact $N_{\mathscr{H}_{\alpha}}\left(\mathcal{U}_{\alpha}\right)=\{0\}$ whatever is $\mathcal{U}_{\alpha}$. In short, (40) contains all the stipulations implied when a contact is declared frictionless.

But, in addition, (40) entails $\mathscr{U}_{\alpha} \in \mathscr{K}_{\alpha}(t, q)$, since otherwise $N_{\mathscr{F}_{\alpha}}\left(\mathscr{U}_{\alpha}\right)$ would be empty. If $f_{\alpha}(t, x)<0$ this imposes no restriction on $U_{\alpha}$ while if $f_{\alpha}(t, q) \geqslant 0$, i.e. $\alpha \in J(t, q)$, this imposes $U_{\alpha} \cdot n^{\alpha} \geqslant 0$. By elementary kinematics, $\mathscr{U}_{\alpha} \cdot n^{\alpha}$ is found equal to the time-derivative of the normal gap between the concerned bodies. Similarly to the 'integration lemma' of Subsection 8.2 , this may be proved to secure that non-interpenetration holds for every $t>t_{0}$, provided it holds at $t_{0}$.

Generally speaking, we say that a package of information, concerning the possible contact labelled $\alpha$, is a complete contact law if, among other phenomenological stipulations, it implies the following:

in all cases $\mathscr{U}_{\alpha} \in \mathscr{K}_{\alpha}$,

if $\mathscr{U}_{\alpha} \in$ interior $\mathscr{K}_{\alpha}$, then $\mathscr{R}^{\alpha}=0$.

Such is the law of frictionless contact in the formulation (40). Concerning similarly the description of non-interpenetrability with Coulomb friction in terms of a complete contact law, see [12,45].

\subsection{The Gauss-Seidel way}

In the algorithmic scheme sketched in Subsection 8.3, if the specificities of the no-friction case are let aside, the phenomenological laws relative to each possible contact have to be introduced. Instead of the concise writing (37), the discretized equation of dynamics has to be detailed in the form

$$
A_{\mathrm{m}}\left(u_{\mathrm{f}}-u_{\mathrm{i}}\right)=h F_{\mathrm{m}}+\sum_{\alpha \in J_{\mathrm{m}}} p^{\alpha}
$$

where the element $p^{\alpha}$ of $\boldsymbol{R}^{n}$ is made of the covariant components of the impulsion at contact $\alpha$, i.e. the integral $\mathscr{P}^{\alpha}$ over $\left[t_{\mathrm{i}}, t_{\mathrm{f}}\right]$ of the contact force $\mathscr{R}^{\alpha}$. Throughout the time-step, one ascribes to the linear mapping $G_{\alpha}^{*}$ of Eq. (27) its value computed at $\left(t_{\mathrm{m}}, q_{\mathrm{m}}\right)$, hence 


$$
p^{\alpha}=G_{\alpha}^{*} \mathscr{P}^{\alpha}
$$

One does the same with the linear mapping $G_{\alpha}$ and with the rheonomic term $\mathscr{W}_{\alpha}$ of Eq. (26). Since a discretization scheme of the implicit type is being planned, the final velocity $u_{\mathrm{f}}$ is invoked, so as to calculate

$$
\mathcal{U}_{\alpha, \mathrm{f}}:=G_{\alpha} u_{\mathrm{f}}+\mathcal{W}_{\alpha}
$$

In the exact problem, the contact law with label $\alpha$ should connect $\mathcal{U}_{\alpha}$ to the contact force $\mathscr{R}^{\alpha}$ at every instant of the interval $\left[t_{\mathrm{i}}, t_{\mathrm{f}}\right]$. Approximation of the implicit type consists in connecting, through the same relationship, the final value $\mathcal{U}_{\alpha, \mathrm{f}}$ of $\mathcal{U}_{\alpha}$ to the average of this force, namely $\mathscr{P}^{\alpha} / h$. In usual situations such as the frictionless contact described by (40) or also Coulomb friction, the contact law happens to be positively homogeneous (with degree one) relatively to the contact force, so the discretized contact law reads

$$
\operatorname{law}_{\alpha}\left(t_{\mathrm{m}}, q_{\mathrm{m}}, \mathscr{U}_{\alpha, \mathrm{f}}, \mathscr{P}^{\alpha}\right)=\text { true. }
$$

Solving the system of conditions (41) to (44) is the hard part of the computation step. From (41) and (42), one obtains

$$
u_{\mathrm{f}}=u_{\mathrm{r}}+A_{\mathrm{m}}^{-1} \sum_{\beta \in J_{\mathrm{m}}} G_{\alpha}^{*} \mathscr{P}^{\beta},
$$

where $u_{\mathrm{r}}:=u_{\mathrm{i}}+h A_{\mathrm{m}}^{-1} F_{\mathrm{m}}$ may be named the relaxed velocity. Here is an iteration technique à la Gauss-Seidel which consists of treating a succession of single-contact problems.

Let an estimated solution ( $u_{\mathrm{f}}^{\text {esti }}, \mathscr{P}_{\text {esti }}^{\beta}$ ), $\beta$ running through $J_{\mathrm{m}}$, be obtained with (45) satisfied. One expects to obtain a corrected estimate, say $\left(u_{\mathrm{f}}^{\text {corr }}, \mathscr{P}_{\text {corr }}^{\beta}\right)$, by selecting a label $\alpha$ and altering only $\mathscr{P}^{\alpha}$, i.e. $\mathscr{P}_{\text {corr }}^{\beta}=\mathscr{P}_{\text {esti }}^{\beta}$ for $\beta \neq \alpha$. The new estimate is astrained to verify (45), i.e. since the old estimate satisfies the same,

$$
u_{\mathrm{f}}^{\text {corr }}=u_{\mathrm{f}}^{\text {esti }}+A_{\mathrm{m}}^{-1} G_{\alpha}^{*}\left(\mathscr{P}_{\text {corr }}^{\alpha}-\mathscr{P}_{\text {esti }}^{\alpha}\right)
$$

and to satisfy the contact law (44). Through applying $G_{\alpha}$ to both members of (46), one gives to (44) the form

$$
\operatorname{law}_{\alpha}\left(t_{\mathrm{m}}, q_{\mathrm{m}}, G_{\alpha} u_{\mathrm{f}}^{\mathrm{esti}}+\mathscr{W}_{\alpha}+H_{\alpha}\left(\mathscr{P}_{\text {corr }}^{\alpha}-\mathscr{P}_{\mathrm{esti}}^{\alpha}\right), \mathscr{P}_{\text {corr }}^{\alpha}\right)=\text { true }
$$

where $H_{\alpha}:=G_{\alpha} A_{\mathrm{m}}^{-1} G_{\alpha}^{*}$ is a symmetric positive definite $3 \times 3$ matrix.

Solving (47) in the unknown $\mathscr{P}_{\text {corr }}^{\alpha}$ is easy in some significant cases, such as two-dimensional Coulomb contact or the case where $H_{\alpha}$ is axissymmetric about $n^{\alpha}$ [42,43]. Generally, some iterative procedures may be applied, in which the normal and tangential components of the contact force are alternatively treated as known. When the normal component is known, Coulomb law assumes the same form as that of 'associated' plasticity, for which a 'principle of maximal dissipation' is known to hold [40]. Anyway, when a technique has been devised for doing this, the above procedure of correcting successive estimates is iterated, with $\alpha$ ranging cyclically in $J_{\mathrm{m}}$. The decision of stopping iterations may be made on observing the magnitude of the vector $\mathscr{P}_{\text {corr }}^{\alpha}-\mathscr{P}_{\text {esti }}^{\alpha}$; this actually is equivalent to checking the precision at which each pair $\left(u_{\mathrm{f}}^{\text {esti }}, \mathscr{P}_{\text {esti }}^{\alpha}\right)$ satisfies the corresponding contact law.

Observe that, provided such a numerical convergence check is made, the linear operator $H_{\alpha}$ in (47) may be replaced by any other mapping of $\boldsymbol{R}^{3}$ into itself with zero limit at point zero, which could have the advantage of making resolution easier. Such a replacement is also used in tricks for accelerating convergence.

The mathematical convergence of algorithms of this sort, in the case of Coulomb contact, as well as the very existence of solutions to the problems addressed, has only been established in special situations [21,29]; uniqueness cannot be expected in general.

If all the contact laws invoked are complete in the sense precised in the foregoing, one sees that, for each $\alpha$, the final relative velocity $\mathscr{U}_{\alpha \mathrm{f}}$ belongs to $\mathscr{K}_{\alpha}\left(t_{\mathrm{m}}, q_{\mathrm{m}}\right)$. Similarly to Subsection 8.3 , Remark 2 , this ensures non-interpenetration with a welcome self-correction effect if the step-length is not too large. Complete contact 
laws also manage automatically the possible breaking of contacts, without referring explicitly to any analysis of complementarity conditions.

\section{Collisions}

Assume that, in the course of the above computation, a time-step exhibits some contacts not detected at the preceding step. This reflects collisions occurring in the system. Since the velocity $u_{\mathrm{f}}$ has to comply with some non-interpenetration conditions from which $u_{\mathrm{i}}$ is exempt, the contact impulsions $p^{\alpha}$ involved in the discretized equation of dynamics (41) are expected to take values of larger magnitude than in the case of a succession of steps with constant $J_{\mathrm{m}}$. But calculation remains the same and delivers kinematically admissible post-collision velocities. In short, because the contact laws (44) have been assumed positively homogeneous in their last arguments, the algorithm treats collisions on the same footing as permanent contacts.

Here is the theoretical model that the algorithm approximates. If discontinuous, the velocity function $u$ is however assumed to have locally bounded variation. Then, at the instant $t_{\mathrm{c}} \in\left[t_{\mathrm{i}}, t_{\mathrm{f}}\right]$ of a possible collision, the left- and right-limit $u^{-}\left(t_{\mathrm{c}}\right)$ and $u^{+}\left(t_{\mathrm{c}}\right)$ exist, considered as approximated in the algorithm by $u_{\mathrm{i}}$ and $u_{\mathrm{f}}$. In the frictionless case of Subsection 8.3, the latter are found connected by Eq. (38). If $h$ is sufficiently small, the term $h F$ may be neglected (recall that proximation mappings are non-expansive). Thus, the collision model one is approximating consists in asserting that $u^{+}\left(t_{\mathrm{c}}\right)$ equals, in the sense of the kinetic metric, the nearest point to $u^{-}\left(t_{\mathrm{c}}\right)$ in the ARVS. Coming back to contact locus, in the notations of Subsection 9.1, this zero-friction calculation in the 'implicit spirit' amounts to admit that, for each $\alpha$, the contact impulsion $\mathscr{P}^{\alpha}$ is connected, through the complete law of frictionless contact (40), with the post-collision velocity $\mathcal{u}_{\alpha}^{+}$. The completeness of the law obviously involves the implication $\mathscr{P}^{\alpha} \neq \mathbf{0} \Rightarrow \mathcal{U}_{\alpha}^{+} \cdot n^{\alpha}=0$, meaning that, if the contact labelled $\alpha$ takes an effective part in the collision process, it is exhibits the feature traditionally qualified as zero restitution.

The present model (it was introduced in [41] under the name of Standard Inelastic Shock) is somewhat more realistic than the traditional one, in that all the contacts present at instant $t_{\mathrm{c}}$ are treated collectively. This produces plausible results, for instance in the case of a slender body rocking on a horizontal plane: if the traditional formulation of zero restitution was applied to all contacts, no rocking could be found.

The energy balance of a collision is mainly of interest in the scleronomic case, i.e. when all the possible external boundaries are fixed. This balance may be drawn by using a rule of the Calculus of functions with bounded variation, namely in the notations of Section 6 ,

$$
\mathrm{d}\|u\|^{2}=\left(u^{+}+u^{-}\right) \cdot \mathrm{d} u .
$$

The norm and scalar product here should naturally be understood in the sense of the kinetic metric (for the energy balance of the possibly nonsmooth motion of the system over a time interval, a more general equality of measures is available $[43,44]$, taking into account the evolution of the matrix $A$ ).

By restricting the measures to the singleton $\left\{t_{c}\right\}$, one retrieves from this formula the energy balance equation sometimes called Kelvin's theorem. This actually is nothing else than the elementary equality $(a+b) .(a-b)=$ $\|a\|^{2}-\|b\|^{2}$. After comparing the balance with the inequalities (22) one concludes that the collisions described by the above model are essentially dissipative.

Many extensions are formally possible. For instance, one may decide to connect through the contact law (40) the contact percussion $\mathscr{P}^{\alpha}$ with the mean velocity $\left(\mathcal{U}_{\alpha}^{-}+\mathscr{U}_{\alpha}^{+}\right) / 2$ : this results in energy-preserving collisions. More generally, some weighted averages of $\mathcal{U}_{\alpha}^{-}$and $\mathcal{U}_{\alpha}^{+}$may be introduced. Similar calculation can also be performed by using, instead of (40) a complete law of frictional contact.

In some definite instances, the results of this sort of computation have been found in very good agreement with experimental observations $[45,46]$, but the preceding should by no means be accepted as a general theory of collisions. In fact, when collisions are analyzed at a finer time-scale, a nonzero micro-duration is assigned to the collision process. The direction of the sliding velocity $\mathcal{u}_{\alpha}$ at the impact locus may be found to exhibit large variations as a function of the micro-time, preventing the identification of any representative velocity which could, phenomenologically, be connected with the total impulsion $\mathscr{P}^{\alpha}$. The situation is better if $\mathcal{U}_{\alpha}$ remains zero: because the Coulomb cone, closed and convex, is constant with regard to the micro-time, the condition of $\mathscr{R}^{\alpha}$ belonging to it commutes with the integration invoked in defining $\mathscr{P}^{\alpha}$. 


\section{References}

[1] J. Abadie, On the Kuhn-Tucker theorem, in: J. Abadie, ed., Nonlinear Programming (North-Holland, Amsterdam, 1967) 17-36.

[2] M. Anitescu and F. A. Potra, Formulating dynamic multi rigid body contact problems with friction as solvable linear complementarity problems, Nonlinear Dyn. 14 (1997) 231-247.

[3] J.P. Aubin and A. Cellina, Differential Inclusions (Springer-Verlag, Berlin, 1984).

[4] D. Baraff, Fast contact force computation for non-penetrating rigid bodies, Computer Graphics (Proc. SIGGRAPH) 28 (1994) $23-34$.

[5] H. Brézis, Opérateurs Maximaux Monotones et Semi-groupes de Contraction dans les Espaces de Hilbert (North-Holland, Amsterdam, 1973).

[6] B. Brogliato, Nonsmooth Impact Mechanics. Models, Dynamics and Control, Lecture Notes in Control and Information Sciences, Vol.220 (Springer-Verlag, Berlin, 1996).

[7] F.H. Clarke, Optimization and Nonsmooth Analysis (Wiley Interscience, New York, 1983).

[8] P.A. Cundall, A computer model for simulating progressive large scale movements of blocky rock systems, Proc. Symposium of the International Society of Rock Mechanics, Vol.1 (Nancy, France, 1971) 132-150.

[9] A. Curnier, A theory of friction, Int. J. Solids Struct. 20 (1984) 637-647.

[10] E. Delassus, Mémoire sur la théorie des liaisons finies unilatérales, Ann. Sci. Ecole Norm. Sup. 34 (1917) $95-179$.

[11] K. Deimling, Multivalued Differential Equations (de Gruyter, Berlin, 1992).

[12] G. de Saxcé and Z. Q. Feng, New inequation and functional for contact with friction, J. Mech. Struct. Machines 19 (1991), $301-325$.

[13] N. Dinculeanu, Vector Measures (Pergamon, London, 1967).

[14] J. Diestel and J.J. Uhl, Vector measures, Math. Surveys 15 (American Math. Soc., Providence RI, 1977).

[15] T.G. Drake and O.R. Walton, Comparison of experimental and simulated grain flows, J. Appl. Mech. 62 (1995) $131-135$.

[16] J. Duran, T. Mazozi, S. Luding, E. Clément and J. Rajchenbach, Discontinuous decompaction of a falling sandpile, Phys. Rev. E 53 (1996) 1923

[17] A. Gavioli, Approximation from the exterior of a multifunction and its application in the 'sweeping' process, J. Diff. Eqns. 92 (1991) $373-383$.

[18] E.J. Haug, Computer Aided Kinematics and Dynamics, Vol. I: Basic Methods (Allyn and Bacon, Boston, 1989).

[19] J.B. Hiriart-Urruty and C. Lemaréchal, Convex Analysis and Minimization Algorithms I (Springer-Verlag, Berlin, etc., 1993).

[20] M. Jean and J. J. Moreau, in: Monuments under Seismic Action, Contract Report, Commission of the European Communities, Environment programme, Contract Nr. EV5V CT93 0300, 1997.

[21] F. Jourdan, P. Alart and M. Jean, A Gauss-Seidel-like algorithm to solve frictional contact problems, Comput. Methods Appl. Mech. Engrg. 155 (1998) 31-47.

[22] A. Klarbring, Mathematical programming and augmented lagrangian methods for frictional contact problems. In: A. Curnier, ed., Proc. Contact Mechanics International Symposium (Presses Polytechniques et Universitaires Romandes, Lausanne, 1992) 409-422.

[23] M. Kunze and M.D.P. Monteiro Marques, Yosida-Moreau regularization of sweeping processes with unbounded variation, J. Diff. Eqns. 130 (1996) 292-306.

[24] M. Kunze and M.D.P. Monteiro Marques, B.V. Solutions to evolution problems with time-dependent domain, Set-valued Anal., 5 (1997) 57-72.

[25] M. Kunze and M.D.P. Monteiro Marques, On the discretization of degenerate sweeping processes, Portugalia Mathematica, to appear.

[26] P. Lötstedt, Mechanical systems of rigid bodies subject to unilateral constraints, SIAM J. Appl. Math. 42 (1982) $281-296$.

[27] M.D.P. Monteiro Marques, Rafle par un convexe semi-continu inférieurement d'intérieur non vide en dimension finie, Comptes Rendus Acad. Sci. Paris 299 Série I (1984) 307-310.

[28] M.D.P. Monteiro Marques, Regularization and graph approximation of a discontinuous evolution problem, J. Diff. Eqns. 67 (1987) 145-164.

[29] M.D.P. Monteiro Marques, Differential Inclusions in Nonsmooth Mechanical Problems: Shocks and Dry Friction (Birkhäuser, Basel, Boston, Berlin, 1993).

[30] J.J. Moreau, Décomposition orthogonale d'un espace hilbertien selon deux cônes mutuellement polaires, Comptes Rendus Acad. Sci. Paris 255 (1962) 238-240.

[31] J.J. Moreau, Les liaisons unilatérales et le principe de Gauss, Comptes Rendus Acad. Sci. Paris 256 (1963) $871-874$.

[32] J.J. Moreau, Proximité et dualité dans un espace hilbertien, Bull. Soc. Math. France, 93 (1965) $273-299$.

[33] J.J. Moreau, Quadratic programming in mechanics: dynamics of one-sided constraints, SIAM J. Control 4 (1966) $153-158$.

[34] J.J. Moreau, Problème d'évolution associé à un convexe mobile d'un espace hilbertien, Comptes Rendus Acad. Sci. Paris 276 (1973) $791-794$.

[35] J.J. Moreau, On unilateral constraints, friction and plasticity. In: G. Capriz and G. Stampacchia, eds., New Variational Techniques in Mathematical Physics (C.I.M.E. II ciclo 1973, Edizioni Cremonese, Roma, 1974) 173-322.

[36] J.J. Moreau, Multiapplications à rétraction finie, Ann. Scuola Norm. Sup. Pisa 1 (1974) 169-203.

[37] J.J. Moreau, Intersection of moving convex sets in a normed space, Math. Scand. 36 (1975) $159-173$.

[38] J.J. Moreau, Evolution problem associated with a moving convex set in a Hilbert space, J. Diff. Eqns. 26 (1977) $347-374$.

[39] J.J. Moreau, Approximation en graphe d'une évolution discontinue, RAIRO Analyse Numérique/Numer. Anal., 12 (1978) $75-84$.

[40] J.J. Moreau, Application of convex analysis to some problems of dry friction. In: H. Zorski, ed., Trends in Applications of Pure Mathematics to Mechanics, Vol. 2 (Pitman Pub. Ltd., London, 1979) 263-280.

[41] J.J. Moreau, Standard inelastic shocks and the dynamics of unilateral constraints. In: G. Del Piero and F. Maceri, eds., Unilateral Problems in Structural Analysis, CISM Courses and Lectures Vol. 288 (Springer-Verlag, Wien, New York, 1985) $173-221$. 
[42] J.J. Moreau, Une formulation du contact à frottement sec; application au calcul numérique, Comptes Rendus Acad. Sci. Paris, Sér.II 302 (1986) 799-801.

[43] J.J. Moreau, Unilateral contact and dry friction in finite freedom dynamics. In: J.J. Moreau and P.D. Panagiotopoulos, eds., Nonsmooth Mechanics and Applications, CISM Courses and Lectures, Vol. 302 (Springer-Verlag, Wien, New York, 1988) 1-82.

[44] J.J. Moreau, Bounded variation in time. In: J.J. Moreau, P.D. Panagiotopoulos and G. Strang, eds., Topics in Nonsmooth Mechanics (Birkhäuser, Basel, Boston, Berlin, 1988) 1-74.

[45] J.J. Moreau, Some numerical methods in multibody dynamics: application to granular materials, Eur. J. Mech., A/Solids 13 Nr.4-suppl.(1994) 93-114.

[46] J.J. Moreau, Numerical experiments in granular dynamics: vibration-induced size segregation. In: M. Raous et al. eds., Contact Mechanics (Plenum Press, New York, 1995) 347-358.

[47] M. Müller, S. Luding and H.J. Herrmann, Simulation of vibrated granular media in two and three dimensional systems, in: P. Grassberger and D. Wolf, eds., Proc. HLRZ-Workshop on Friction, Arching, Contact Dynamics (World Scientific, Singapore, 1997) 335-340.

[48] J.T. Oden and J.A.C. Martins, Models and computational methods for dynamic friction phenomena, Comput. Methods Appl. Mech. Engrg. 52 (1985) 527-634.

[49] L. Paoli and M. Schatzman, Mouvements à un nombre fini de degrés de liberté avec contraintes unilatérales: cas avec perte d'énergie, Math. Model. Numer. Anal. 27 (1993) 673-717.

[50] F. Pfeiffer and C. Glocker, Multibody Dynamics with Unilateral Contacts (John Wiley and Sons, New York, 1996).

[51] R.T. Rockafellar, Convex Analysis (Princeton University Press, Princeton, 1970).

[52] A. Signorini, Sopra alcune questioni di elastostatica, Atti Soc. Ital. per il progresso delle Scienze, 1933.

[53] D.E. Stewart and J.C. Trinkle, An implicit time-stepping scheme for rigid body dynamics with inelastic collisions and Coulomb friction, Int. J. Numer. Methods Engrg. 39 (1996) 2673-2691.

[54] D.E. Stewart, Existence of solutions to rigid body dynamics and the Painlevé paradoxes, Comptes Rendus Acad. Sci. Paris 325 Série I (1997) 689-693.

[55] S.P. Stoianovici and Y. Hurmuzlu, A critical study of the concepts of rigid body collision theory, J. Appl. Mech. 63 (1996) 307-316.

[56] J. Trinkle, J.-S. Pang, S. Sudarski and G. Lo, On dynamic multi-rigid-body contact problems with Coulomb friction, Zeitschrift für Angewandte Mathematik und Mechanik 77 (1997) 267-279.

[57] M. Valadier, Entrainement unilatéral, lignes de descente, fonctions lipschitziennes non pathologiques, Comptes Rendus Acad. Sci. Paris 308 Série I (1989) 241-244.

[58] M. Valadier, Lipschitz approximations of the sweeping (or Moreau) process, J. Diff. Eqns. 88 (1990) 248-264.

[59] O.R. Walton, Application of molecular dynamics to macroscopic particles, Int. J. Engrg. Sci. 22 (1984) 1097-1107. 